\title{
Noise-free Latent Block Model for High Dimensional Data
}

\author{
Charlotte Laclau • Vincent Brault
}

Received: date / Accepted: date

\begin{abstract}
Co-clustering is known to be a very powerful and efficient approach in unsupervised learning because of its ability to partition data based on both the observations and the variables of a given dataset. However, in high-dimensional context co-clustering methods may fail to provide a meaningful result due to the presence of noisy and/or irrelevant features. In this paper, we tackle this issue by proposing a novel co-clustering model which assumes the existence of a noise cluster, that contains all irrelevant features. A variational expectation-maximization (VEM)-based algorithm is derived for this task, where the automatic variable selection as well as the joint clustering of objects and variables are achieved via a Bayesian framework. Experimental results on synthetic datasets show the efficiency of our model in the context of high-dimensional noisy data. Finally, we highlight the interest of the approach on two real datasets which goal is to study genetic diversity across the world.
\end{abstract}

\section{Introduction}

Clustering, which aims to partition data into groups (clusters) of similar objects, has a wide range of applications including information retrieval, bioinformatics, pattern recognition and image analysis. In many of these cases, and particularly in the case of high dimensional data, a significant proportion of the variables is not providing any relevant information. In this situation, attempting to learn while including this part of the data, which can be qualified as noise, strongly disrupts the clustering algorithms and can mask the existing structure. Despite the need for a theoretical and practical framework, where one can consider only

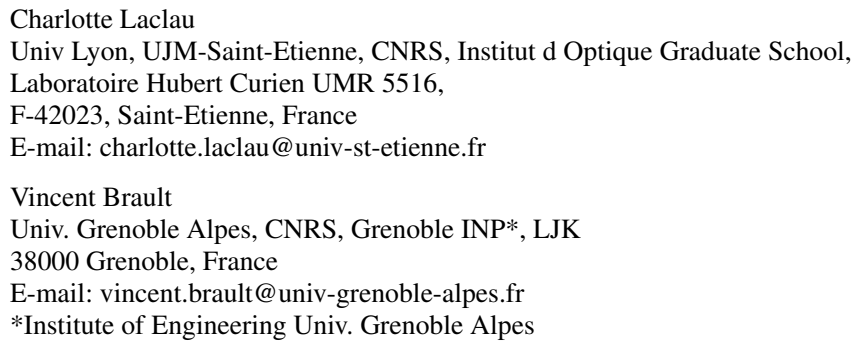


the subset of relevant variables for partitioning the data, relatively little work has been proposed so far in this direction. Noise management remains a complex issue, which raises the question of its definition and, consequently, its modelling. Indeed, in unsupervised learning, where there is no labels to guide this search, one can define the notion of noise in many different ways. For example, in genetic data analysis, the common approach to handle noise is to eliminate genes with a low variance, that is, with a homogeneous degree of expression across all individuals. This pre-processing step (or filtering) relies on the intrinsic properties of the variables to determine their relevance but completely ignores the possible interactions between the variables and the structure, and between the variables themselves. Other approaches have sought to weight the variables according to their discriminating power and to learn clusters simultaneously. These approaches are generally more efficient than the socalled filtering methods, but the weight calculation for each variable induces an algorithmic complexity which makes them impracticable in the context of high-dimensional data.

In the context of outlier detection, Dave $(1991,1993)$ and more recently Ben-David and Haghtalab (2014) proposed a novel formalism that allows to transform clustering algorithms, based on the notion of centroids (and hence distance), into robust algorithms for noisy objects. Their approach is based on the interesting concept of the existence of a potential noise cluster, i.e., a cluster that contains the set of noisy objects, without specifying or constraining them to be similar. Despite encouraging results, this type of approach has not been extended to the problem of noise variables and has remained limited to the framework of some metric approaches. To overcome these limitation, and to address the problem of noise cluster from the variable perspective, we propose to exploit the framework of co-clustering (or bi-clustering) (Hartigan, 1972; Mirkin, 1996), which aims to simultaneously cluster the sets of objects and variables into homogeneous blocks (or co-clusters). These blocks consist of subset of the data matrix composed of objects and variables strongly linked. In some sense, one can see co-clustering as a local variable selection approach which benefits from the knowledge of the object partition. Furthermore, in order to cover different aspects of the definition of noise, we propose a probabilistic approach, allowing a more flexible modelling of the noise. To this end, we assume that the data are generated according to a mixture consisting in the cartesian product of two probability densities associated with different subsets of variables (Law et al, 2004): parameters of the first one are independent from the structure, while parameters of the second one are specific to each blocks.

As a result, our contributions can be summarized as follows:

- We design a novel probabilistic co-clustering model which relies on the assumption that there exists a variable cluster that contains only irrelevant features, referred to as the "noise" variable cluster, in the following. All the variables belonging to this cluster are assumed to be drawn from a probability distribution that does not depend on the structure of the data into groups. Then, for all observations and the remaining variables, we extract relevant partitions, which provide a clear interpretation of the data structure.

- The optimization of the model is carried out by a Variational EM-based (VEM) algorithm. In addition, we propose a Bayesian version and introduce Gibbs sampling on the different parameters to overcome the problem of vanishing clusters.

- In unsupervised learning, the estimation of the number of clusters is also a key point. To this end, we propose to adapt a model selection criteria, namely the Integrated Completed Likelihood (ICL) (Biernacki et al, 2000; Keribin et al, 2014).

For all the contributions mentioned above, we provide theoretical guarantees on the identifiability and the consistency of our model. Finally, we extensively validate our approach 
over synthetic datasets and study the relevance of the proposed model over a real dataset on genetic diversity.

The remainder of this paper is organized as follows. First, we give a brief overview of related works that tackle the problem of clustering and feature selection using modelbased approaches in Section 2. We proceed by formally defining an appropriate latent block model for simultaneous co-clustering and feature selection, named Noise-Free Latent Block Model (NFLBM) in Section 3. Section 4 describes three different optimization procedures, all derived from the Variational EM algorithm (VEM). In Section 5, we adapt the ICL criteria to the NFLBM and give an explicit formulation for its calculation. In Section 6, we provide a detailed theoretical analysis of the proposed model. Then, Section 7 illustrates the ability of our approach to identify irrelevant features on synthetic and one genetic dataset. We conclude this paper by summarizing the contributions and discussing possible perspectives of this work in Section 8.

\section{Preliminary knowledge}

This section gives an overview of clustering approaches which aim at performing either object or feature selection in the model-based framework. Also, we provide a description of the Latent Block Model (LBM), a probabilistic model for co-clustering which we use to develop our approach.

\subsection{Clustering on noisy data}

Noise-cluster and robust clustering. Several directions have been taken in developing robust clustering algorithms (Dave, 1991, 1993; Cuesta-Albertos et al, 1997; Ester et al, 1996; Ben-David and Haghtalab, 2014). The concept of noise cluster was first introduced by Dave $(1991,1993)$ in a fuzzy centroid-based setting where assume the existence of a fictitious cluster which prototype (or center) is equidistant from all the objects of the dataset. Then objects which are further away from all other centers are assigned to this center and denoted as part of the noise cluster. Cuesta-Albertos et al (1997) proposed to use the concept of trimming in order to determine the subset of objects, of a predefined fixed size, whose removal leads to the maximum improvement of the objective function of k-means, and therefore of the clustering quality. They also extend the concept of trimming and provide strong theoretical guarantees for this family of approaches using influence functions (García-Escudero et al, 2008). Another family of approaches was explored by Ester et al (1996) to introduce the notion of noise cluster. They developed a density-based approach for clustering noisy objects where all objects in the dataset which belong to the sparse regions are assigned to the noise cluster. This concept of noise cluster was further extended and theoretically formalized by Ben-David and Haghtalab (2014), who proposed to generalize all these approaches to any prototype-based algorithms. For more references on robust clustering algorithms, we refer the reader to the survey of García-Escudero et al (2010).

The aforementioned approaches focus on the problem of noisy objects, or outliers, which generally represent a small proportion of the data. In this work, we aim to deal with highdimensional data, therefore our goal is to target noisy features and to extend the concept of noisy cluster to the problem of feature selection. In addition, we propose to explore this concept in the probabilistic framework of mixture models, allowing more flexibility regarding the modelling of noise. 
Model-based feature selection. Several work have been conducted in order to perform feature selection using the framework of mixture models. These approaches can be broadly divided into three categories. The first proposes to cast the problem as a model selection problem, and includes the work of Raftery and Dean (2006), Maugis et al (2009) and Celeux et al (2011). For instance, Raftery and Dean (2006) proposed to divide the initial set of variables into two groups: a set containing relevant features and a set containing irrelevant features, that are assumed to be dependent according to a linear relationship. Models in competition are compared based on their integrated log-likelihood and aim to maximize a two terms criterion, where the first term corresponds to the BIC resulting from the clustering on the set of relevant features while the second term relies on the linear regression of irrelevant variables on the set of relevant variables.

Another attempt to perform feature selection and clustering is to progressively add sparsity in the features by penalizing the log-likelihood function to optimize. Pan and Shen (2007) proposed a penalized clustering model for continuous data by considering a $\ell_{1}$ penalty function focused on the mean of each cluster. The idea behind this approach is to define a feature as irrelevant if its mean is equal in all components. In the same line of reasoning several articles adapted or extended this concept of penalized model with variable selection (Wang and Zhu, 2008; Zhou et al, 2009).

A third way to perform feature selection in the model-based framework was introduced in (Law et al, 2004). The authors considered a Gaussian mixture model and decomposed the Normal distribution, commonly used in this case, into a product of two probability distributions. Parameters of the first distribution are specific to each cluster while parameters of the second distribution are independent from the clustering partition. This approach was further extended to other types of distributions (Wang and Kabán, 2005; Li and Zhang, 2008). The main drawbacks of these models is that even though they reduce the impact of the irrelevant features on the partition of the data, by assigning to them a low weight, they still consider them while computing the clustering partitions. For more details on model-based feature selection, we refer the reader to the survey of Bouveyron and Brunet-Saumard (2014).

Despite the good results for identifying irrelevant features in simple clustering, these methods present an increasing computational complexity with respect to the number of features, which make them impracticable in the context of high-dimensional data. Starting from the original idea of Law et al (2004), we propose a novel model, that aims to tackle the aforementioned problems. First, we extend their one-way clustering approach to co-clustering, which is known to be more efficient than simple clustering for high-dimensional data, and specifically for data where the number of features is much larger than the number of observations. Furthermore, in (Law et al, 2004), the authors do not truly consider feature selection but rather feature weighting. Indeed, they estimate the so-called feature saliency for each feature given by the probability that it is relevant. Therefore, features with a higher saliency will have more weight on the final partition of the data observations, but all features, including the noisy ones, will have an impact on this latter too; in our model, we propose to exclude irrelevant features and to isolate them in a noise cluster. Finally, from a computational point of view, our model replaces the estimation of a number of parameters equal to the number of features by only one parameter that indicates the proportions of noisy features, making it more suitable for high-dimensional data. 


\subsection{Latent block models}

Notations. We assume that the data are represented by a matrix $\mathbf{x}=\left\{x_{i j}, i \in I=\right.$ $\{1, \ldots, n\} ; j \in J=\{1, \ldots, d\}$ ), where $n$ and $d$ denote the number of objects and variables, respectively. In this work we will only provide the mathematical derivations for binary matrices (as the generalization to categorical data is straightforward), i.e. $x_{i j} \in\{0,1\}$. A partition of $I$ into $g$ clusters is represented by a label vector $\mathbf{z}=\left\{z_{1}, \ldots, z_{n}\right\}$, where $\mathbf{z}_{i} \in\{1, \ldots, g\}$. In a similar way, we define the partition of $J$ into $m$ clusters by $\mathbf{w}=\left\{w_{1}, \ldots, w_{d}\right\}$, where $\mathbf{w}_{j} \in\{1, \ldots, m\}$. The partitions of $I$ (resp. $J$ ) can also be represented by a classification matrix of elements in $\{0,1\}^{g}$ (resp. $\{0,1\}^{m}$ ) such that $\mathbf{z}=\left(z_{i k}, i=1, \ldots, n, k=1, \ldots, g\right)$ $\left(\right.$ resp. $\left.\mathbf{w}=\left(w_{j \ell}, j=1, \ldots, d, \ell=1, \ldots, m\right)\right)$, where $z_{i k}=1$ (resp. $\left.w_{j \ell}=1\right)$ if element $i$ (resp. $j$ ) belongs to cluster $k$ (resp. $\ell$ ), and 0 otherwise.

Finally, sums and products related to rows, columns, row's cluster and column's cluster are subscripted by the letters $i, j, k$ and $\ell$ without indicating the limits of variation which will be implicit. So, the sums $\sum_{i}, \sum_{j}, \sum_{k}$ and $\sum_{\ell}$ stand for $\sum_{i=1}^{n}, \sum_{j=1}^{d}, \sum_{k=1}^{g}$ and $\sum_{\ell=1}^{m}$, respectively.

Latent Block Model. The co-clustering task can be embedded into a probabilistic framework, with the Latent Block Model (LBM), proposed by Govaert and Nadif (2003). Given a matrix $\mathbf{x} \in \mathbb{R}^{n \times d}$, this model considered that the univariate random variables $x_{i j}$ are conditionally independent knowing $\mathbf{z}$ and $\mathbf{w}$, with parametrized probability density function (pdf) $f\left(x_{i j} ; \alpha_{k \ell}\right)$ if the row $i$ belongs to the cluster $k$ and the column $j$ belongs to the cluster $\ell$. The conditional pdf of $\mathbf{x}$ knowing $\mathbf{z}$ and $\mathbf{w}$ can be expressed as

$$
\prod_{i, j} f\left(x_{i j} ; \alpha_{z_{i} w_{j}}\right)=\prod_{i, j, k, \ell}\left\{f\left(x_{i j} ; \alpha_{k \ell}\right)\right\}^{z_{i k} w_{j \ell}} .
$$

In this case, the two sets $I$ and $J$ are assumed to be random samples so that the row and column labels become latent variables. This model is based on the following assumptions:

- Conditional independence defined before;

- Independent latent variables: the partitions $\mathbf{z}_{1}, \ldots, \mathbf{z}_{n}, \mathbf{w}_{1}, \ldots, \mathbf{w}_{d}$ are considered as latent variables and assumed to be independent:

$$
p(\mathbf{z}, \mathbf{w})=p(\mathbf{z}) p(\mathbf{w}), \quad p(\mathbf{z})=\prod_{i} p\left(z_{i}\right) \quad \text { and } \quad p(\mathbf{w})=\prod_{j} p\left(w_{j}\right) .
$$

- For all $i$, the distribution of $p\left(z_{i}\right)$ is the multinomial distribution $\mathcal{M}\left(1 ; \pi_{1}, \ldots, \pi_{g}\right)$ and does not depend on $i$. Similarly, for all $j$, the distribution of $p\left(w_{j}\right)$ is the multinomial distribution $\mathcal{M}\left(1 ; \tau_{1}, \ldots, \tau_{m}\right)$ and does not depend on $j$.

The set parameter of the latent block model is denoted by $\boldsymbol{\theta}=(\boldsymbol{\pi}, \boldsymbol{\tau}, \boldsymbol{\alpha})$, where $\boldsymbol{\pi}=$ $\left(\pi_{1}, \ldots, \pi_{g}\right)$ and $\boldsymbol{\tau}=\left(\tau_{1}, \ldots, \tau_{m}\right)$ represent the mixing proportions and $\boldsymbol{\alpha}_{k \ell}$ is the parameter of the distribution for the block $(k, \ell)$.

The LBM can be easily adapted to data of different natures such as continuous, binary or discrete by considering appropriates distributions, like the Gaussian (Govaert and Nadif, 2013), Bernoulli and Multinomial (Keribin et al, 2014) ones. In all cases, the estimation of both partitions $\mathbf{z}$ and $\mathbf{w}$ and the set of parameters of the model is achieved by a Variational EM algorithm.

In real-world application, one can often encounter rows (i.e. objects) or columns (i.e. features) which are irrelevant, and by taking them into account, LBM usually creates a 
very high number of artificial blocks; for instance, by dividing relevant blocks to multiple ones based on noise. In this paper, we propose to overcome this over-learning problem by assuming, in the LBM, that there exists a variable noise cluster which contains all irrelevant features and that these features have no impact on the object clustering.

\section{Noise-free Latent Block Model}

In this section, we describe the modelling assumptions behind the proposed model. As an illustrative example, let us consider the United State Congressional Voting Records data ${ }^{1}$. This dataset contains votes of the members of the U.S. House of Representatives on various political issues. Each element is therefore coded by 2 for "yea", 0 for "nay" and 1 for abstention. In analyzing these data, our goal is threefold.

1. We aim to determine the underlying political groups within the Congress, i.e., we assume $g$ latent groups over the members.

2. We also aim to find the underlying political topics among the voted laws, i.e., we assume $m$ latent groups over the political issues.

3. We aim to differentiate between relevant from non-informative political issues by assuming a set of laws for which the vote will not be influenced by the political group.

Consequently, we assume that the observed data matrix is generated according a mixture where we can decompose the density function into a product of two terms: the first one is associated to a relevant block structure while the second one contains only the irrelevant features and is therefore independent from the latent structure. Then, each member (resp. political issue) is associated with a random vector $\pi_{i}$ (resp. $\tau_{j}$ ), where $\pi_{i k}$ (resp. $\tau_{j \ell}$ ) is the probability of a member $i$ (resp. an issue $j$ ) belonging to group $k$ (resp. $\ell$ ). For each member and issue, the indicator vectors $\left(z_{1}, \ldots, z_{n}\right)$ where $z_{i} \in\{1, \ldots, k, \ldots, g\}$, and $\left(w_{1}, \ldots, w_{d}\right)$ where $w_{j} \in\{1, \ldots, \ell, \ldots, m\}$ represent the group membership of the $i$-th member and the $j$-th issue, respectively.

In addition, in order to deal with irrelevant features, our model relies on the idea that only a proportion $\phi$ of the laws are discriminant, and that the remaining proportion $(1-\phi)$ of are just noise. To proceed we assume the existence of a noise cluster $w_{0}$ and introduce a novel parameter $\phi$ which role is to measure the proportion of relevant features. As a result, we now consider that $\mathbf{w}$ is drawn according to $\prod_{j=1}^{d} \mathcal{M}(1 ;(1-\phi), \phi \boldsymbol{\tau})$ in $\{0, \ldots, m\}$.

On the one hand, features that belong to the noise cluster are assumed to be drawn according to a probability distribution, with parameters $\boldsymbol{\lambda}=\left(\lambda_{j}\right) ; j=1, \ldots, w_{+0}$, where $w_{+0}$ is the number of features in the noise cluster. One can observe that $\lambda$ does not depend on neither $k$ or $\ell$ but is feature-specific, allowing to model different type of noise. For instance, a very small $\lambda_{j}$ indicates that the noisy feature $j$ mostly takes the value 0 , while a high value represents a noisy feature with a majority of 1's. On the other hand, relevant features are assumed to be drawn from a probability distribution with parameters $\boldsymbol{\alpha}=\left(\alpha_{k \ell}\right) ; k=$ $1, \ldots, g ; \ell=1, \ldots, m$, which are block-specific.

In this work, we limit ourselves to the case of Bernoulli distribution, for the sake of clarity, but the extension to other density function (such as Gaussian or Multinomial), and therefore to other types of data (continuous or categorical) is straightforward.

Now, putting everything together we can define the Noise-free Latent Block Model (NFLBM) which postulates that a data matrix $\mathrm{x}$ is drawn from the following generative procedure.

\footnotetext{
1 https://archive.ics.uci.edu/ml/datasets.html
} 


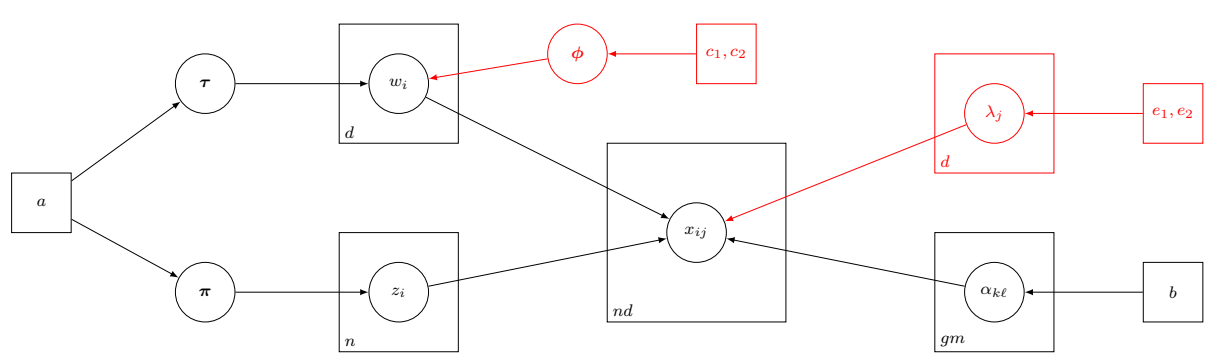

Fig. 1: Bayesian version of the Noise-free Latent Block Model (NFLBM). Elements in red represent the differences w.r.t. the classic LBM for Bernoulli distribution.

- Generating z such that $\forall z, z_{i} \sim \mathcal{M}(1 ; \boldsymbol{\pi})$ in $\{1, \ldots, g\}$.

- Generating w such that $\forall w, w_{j} \sim \mathcal{M}(1 ;(1-\phi), \phi \boldsymbol{\tau})$ in $\{0, \ldots, m\}$.

- Generating x with for each $j \in\{1, \ldots, d\}$ :

- if $w_{j 0}=1, x_{. j} \sim \mathcal{B}\left(\lambda_{j}\right)^{n}$,

- else $\forall x, x_{i j} \sim \mathcal{B}\left(\alpha_{z_{i} w_{j}}\right)$

This process is illustrated as a graphical model in Figure 1. The hyper-parameters related to a Bayesian framework are explained in the next section. The mixture density can be expressed as

$$
\begin{aligned}
p(\mathbf{x} ; \theta) & =\sum_{z, w} p(\mathbf{x} \mid \mathbf{z}, \mathbf{w} ; \theta) p(\mathbf{z}, \mathbf{w} ; \theta) \\
= & \sum_{z, w} \prod_{i, k} \pi_{k}^{z_{i k}} \times(1-\phi)^{w_{+0}} \phi^{\left(d-w_{+0}\right)} \prod_{j, \ell} \tau_{\ell}^{w_{j \ell}} \times \prod_{i, j} f\left(x_{i j}, \lambda_{j}\right)^{w_{j 0}} \times \prod_{i, j, k, \ell} f\left(x_{i j}, \alpha_{k \ell}\right)^{z_{i k} w_{j \ell},}
\end{aligned}
$$

where $w_{+0}$ denotes the number of features that belong to the noise cluster (i.e. the irrelevant features $), f\left(x_{i j}, \alpha_{k \ell}\right)=\alpha_{k \ell}^{x_{i j}}\left(1-\alpha_{k \ell}\right)^{\left(1-x_{i j}\right)}, f\left(x_{i j}, \lambda_{j}\right)=\lambda_{j}^{x_{i j}}\left(1-\lambda_{j}\right)^{\left(1-x_{i j}\right)}$ and $\boldsymbol{\theta}=(\boldsymbol{\pi}, \boldsymbol{\phi}, \boldsymbol{\tau}, \boldsymbol{\lambda}, \boldsymbol{\alpha})$ is the set of parameters of the model.

\section{Parameter Estimation and Posterior Inference}

Now, the goal is to compute the latent variables $\mathbf{z}$ and $\mathbf{w}$ and to estimate the set of parameters, $\theta$. To proceed, one need to maximize the likelihood associated with our model, given by

$$
\begin{aligned}
\mathcal{L}(\theta) & =\sum_{(z, w) \in \mathcal{Z} \times \mathcal{W}} p(\mathbf{z} ; \theta) p(\mathbf{w} ; \theta) p(\mathbf{x} \mid \mathbf{z}, \mathbf{w} ; \boldsymbol{\theta}) \\
& =\sum_{(z, w) \in \mathcal{Z} \times \mathcal{W}}(\underbrace{\prod_{i, k} \pi_{k}^{z_{i k}} \times(1-\phi)^{w_{+0}} \phi^{\left(d-w_{+0}\right)} \prod_{j, \ell} \tau_{\ell}^{w_{j \ell}}}_{A}
\end{aligned}
$$




$$
\times \underbrace{\prod_{j}\left(\lambda_{j}^{x_{+j}}\left(1-\lambda_{j}\right)^{\left(n-x_{+j}\right)}\right)^{w_{j 0}}}_{B} \times \underbrace{\prod_{i, j, k, \ell}\left(\alpha_{k \ell}^{x_{i j}}\left(1-\alpha_{k \ell}\right)^{\left(1-x_{i j}\right)}\right)^{z_{i k} w_{j \ell}}}_{C}),
$$

where $x_{+j}=\sum_{i=1}^{n} x_{i j}$.

The direct optimization of the likelihood for LBM's is a well-known issue: first it is intractable for large dataset, second deriving formula for the latent variables using a classic EM is challenging. To overcome this, Govaert and Nadif (2008) suggests to rather consider the maximization of the variational approximation of the likelihood, namely the Free Energy.

\subsection{VE-step}

The VE (Variational Estimation)-step relies on the computation of the conditional expectation of the complete log-likelihood.

First, we compute the probability for each object to belong to one of the row clusters $k=1, \ldots, g$ :

$$
s_{i k}=\frac{\pi_{k} \prod_{\ell=1}^{m} \prod_{j=1}^{d}\left[f\left(x_{i j}, \alpha_{k \ell}\right)\right]^{t_{j \ell}}}{\sum_{k^{\prime}} \pi_{k^{\prime}} \prod_{\ell=1}^{m} \prod_{j=1}^{d}\left[f\left(x_{i j}, \alpha_{k^{\prime} \ell}\right)\right]^{t_{j \ell}}},
$$

where $s_{i k}=\mathbb{P}\left(z_{i k}=1 ; x, \theta\right)$.

Then, we compute the probability for each feature to belong to one of the column clusters $\ell=1, \ldots, m$ :

$$
t_{j \ell}=\frac{\phi \tau_{\ell} \prod_{i, k}\left[f\left(x_{i j}, \alpha_{k \ell}\right)\right]^{s_{i k}}}{\sum_{\ell^{\prime}=1}^{m} \phi \tau_{\ell^{\prime}} \prod_{i, k}\left[f\left(x_{i j}, \alpha_{k \ell^{\prime}}\right)\right]^{s_{i k}}+(1-\phi) \prod_{i}\left[f\left(x_{i j}, \lambda_{j}\right)\right]},
$$

where $t_{j \ell}=\mathbb{P}\left(w_{j \ell}=1 ; x, \theta\right)$. Finally, we distinguish one column cluster from the other ones which should only contain irrelevant features, i.e., non-discriminant ones:

$$
t_{j 0}=\frac{(1-\phi) \prod_{i}\left[f\left(x_{i j}, \lambda_{j}\right)\right]}{\sum_{\ell^{\prime}=1}^{m} \phi \tau_{\ell^{\prime}} \prod_{i, k}\left[f\left(x_{i j}, \alpha_{k \ell^{\prime}}\right)\right]^{s_{i k}}+(1-\phi) \prod_{i}\left[f\left(x_{i j}, \lambda_{j}\right)\right]}
$$

where $t_{j 0}=\mathbb{P}\left(w_{j 0}=1 \mid \mathbf{x}, \boldsymbol{\theta}\right)$.

One should note that, compared to the classic LBM, the probabilities $s_{i k}$ 's only depend on the relevant column clusters, while the features contained in the noise cluster have no direct impact on their computations. In addition, the $t_{j} \ell$ ' depends of $\phi$, which control the proportion of relevant features. However, when $\phi=1$, i.e., all the features are relevant, then this step boils down to the VE step of a traditional LBM.

\subsection{M-step}

From $B$ and $C$ (equation 1), we obtain the estimators for the parameters of the Bernoulli densities given by

$$
\hat{\lambda_{j}}=\frac{\sum_{i} x_{i j}}{n}=\frac{x_{+j}}{n}, \quad \hat{\alpha}_{k \ell}=\frac{\sum_{i, j} s_{i k} t_{j \ell} x_{i j}}{s_{+k} t_{+\ell}},
$$


where $s_{+k}=\sum_{i} s_{i k}$ and $t_{+\ell}=\sum_{j} t_{j \ell}$. Furthermore, for the variable $j, \lambda_{j}$ consists of elements drawn from the same Bernoulli distribution, which is independent from the latent structure; therefore, its estimation is also independent from the partitions estimation and is solely a statistic of the data.

From $A$ (equation 1), and s.t. $\sum_{k} \pi_{k}=1, \sum_{\ell=0}^{m} \tau_{\ell}=1$, we obtain the proportion of row and column clusters,

$$
\hat{\pi}_{k}=\frac{s_{+k}}{n}, \quad \hat{\tau}_{\ell}=\frac{t_{+\ell}}{d-t_{+0}} \quad \text { and } \quad \hat{\phi}=\frac{d-t_{+0}}{d},
$$

where $t_{+0}=\sum_{j} t_{j 0}$.

We observe that, compared to the M-step of the algorithm for LBM, we have two additional equations for the computation of $\phi$ and $\lambda$. As for the other parameters, their computation is equivalent to that of $\mathrm{LBM}$ when $\phi=1$.

\subsection{Bayesian Inference}

While VEM is known to be an accurate way of estimating the parameters of the latent block models, one of the issue is its tendency to empty small clusters. To overcome this drawback, we also propose a Bayesian version of our framework.

A priori assumptions. In a Bayesian perspective, one can consider proper and independent non informative prior distributions for the mixing proportions $\pi, \tau$, and for the parameters $\boldsymbol{\alpha}$ and $\boldsymbol{\lambda}$ as a product of $g \times m$ and $w_{+0}$ non informative priors on each Bernoulli parameter, respectively. Therefore, for the mixing proportions we have that $\pi, \tau \sim \operatorname{Dir}(a, \ldots, a)$ (where Dir stands for the Dirichlet distribution) that is

$$
p(\boldsymbol{\pi}) \propto \prod_{k=1}^{g} \pi_{k}^{a-1} \quad \text { and } \quad p(\boldsymbol{\tau}) \propto \prod_{\ell=1}^{m} \tau_{\ell}^{a-1},
$$

and for the Bernoulli parameters, we have $\alpha \sim \mathcal{B} e(b, b)$ (where $\mathcal{B} e$ stands for the Beta distribution), $\boldsymbol{\lambda} \sim \mathcal{B} e\left(e_{1}, e_{2}\right)$, that is

$$
p(\boldsymbol{\alpha}) \propto \prod_{k, \ell} \alpha_{k \ell}^{b-1}\left(1-\alpha_{k \ell}\right)^{b-1} \quad \text { and } \quad p(\boldsymbol{\lambda}) \propto \prod_{j: w_{j 0}=1} \lambda_{j}^{e_{1}-1}\left(1-\lambda_{j}\right)^{e_{2}-1} .
$$

Finally, we also assume a priori on the parameter $\phi$

$$
\phi \sim \mathcal{B} e\left(c_{1}, c_{2}\right) \text { i.e. } \quad p(\phi) \propto \phi^{c_{1}-1}(1-\phi)^{c_{2}-1} .
$$

One can observe that we choose two different hyper-parameters for the Beta distributions associated to the $\lambda$ 's and $\phi$ 's parameters, while we set them to be the same for the $\alpha$ 's. This choice and the values of these hyper-parameters will be discussed below. The graphical representation of the Bayesian version of the NFLBM is presented in Figure 1.

In practice, one usually consider Jeffrey's or uniform's prior distributions on the parameters. The problem is that in the case of proportions parameters (i.e. $\pi$ and $\boldsymbol{\tau}$ ) considering these types of distributions usually tends to empty some of the clusters (Frühwirth-Schnatter, 2011). To overcome this, Keribin et al (2014) have shown that taking $a=4$ and $b=1$ allows to prevent cluster from vanishing during the label assignment. For the parameters $\left(e_{1}, e_{2}\right)$ we choose to use uniform distribution so as not to put any type of a priori information, and 
the effect of $\left(c_{1}, c_{2}\right)$ is studied in Section 7. However, one should note that we can induce some specific behaviors depending on the availability of such a priori knowledge on the data:

- On the distribution of $\phi$, we can introduce asymmetry if we have information about the proportion of irrelevant features. For instance, considering $c_{2} \gg c_{1}$ implies that the proportion of noise is important compared to the proportion of relevant features.

- On the distribution of $\boldsymbol{\lambda}$, we can also decide to model irrelevant features in different ways. On the one hand, we can consider that a feature is irrelevant if all the objects have a similar value for this latter, and in this case taking $e_{1}$ and $e_{2}$ smaller than 1 will encourage the $\lambda^{\prime} s$ to be close to the extremes 0 or 1 . On the other hand, if we define noise as features with very mitigates values, taking $e_{1}=e_{2}$ with high values tends to encourage $\lambda$ 's close to 0.5 .

Next, we present the new update formula for all parameters in this Bayesian framework.

New parameter estimation. Let us recall that we now consider the optimization of the Bayes formula given by

$$
p(\theta \mid \mathbf{x}) \propto p(\mathbf{x} \mid \theta) p(\theta) .
$$

From this formula, we can directly derive an EM algorithm for the computation of the MAP estimates.

- The E-Step remains the same than the one previously defined in the NFLBM and therefore the formula for computing the conditional probabilities of the labels are unchanged.

- The M-Step differs as the objective to maximize is augmented by the prior density. The Bayesian version combined with the variational approximation leads to

$$
\hat{\pi}_{k}=\frac{s_{+k}+a-1}{n+g(a-1)}, \quad \hat{\tau}_{\ell}=\frac{t_{+\ell}+a-1}{d-t_{+0}+m(a-1)},
$$

where $s_{+k}, t_{+\ell}$ and $t_{+0}$ are the same than the ones defined in the variational approximation. For the parameters of the Bernoulli densities and the estimate of $\phi$ we have

$$
\hat{\lambda_{j}}=\frac{x_{+j}+e_{1}-1}{n+e_{1}+e_{2}-2}, \quad \hat{\alpha}_{k \ell}=\frac{\sum_{i, j} s_{i k} t_{j \ell} x_{i j}+b-1}{\sum_{i, j} s_{i k} t_{j \ell}+2(b-1)}, \quad \hat{\phi}=\frac{d-t_{+0}+c_{1}-1}{d+c_{1}+c_{2}-2} .
$$

Now, going back to the U.S. votes data, we applied the aforementioned Bayesian version of the NFLBM (for which the extension to the multinomial case is straightforward). The number of co-clusters is automatically determined by an appropriate model selection criteria defined in the next section. In this context, we obtained 5 clusters for the member of the congress and 5 clusters for the political issues (including the noise cluster). This structure is presented in Figure 2. From this figure, one can see a clear block structure for some of the political issues, where groups of congress members voted homogeneously 'yeah' or 'nay'. The noise cluster contains two of the laws (left to the red line).

In addition, we have an information regarding the official political group of each member; we know that among them 168 are labeled as Republicans and the remaining 267 as Democrats. By taking a closer look at the distribution of Republicans and Democrats among the clusters, we also note that cluster 2 is mainly composed of Republicans while clusters 4 and 5 are dominated by Democrats. Clusters 1 and 3 have a mixed representation of both, and therefore we can say that for these members, the vote was not clearly impacted by the 


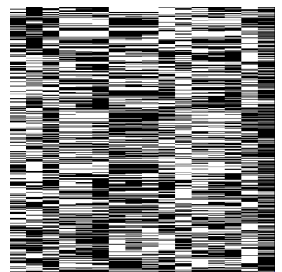

(a)

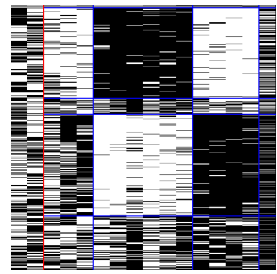

(b)

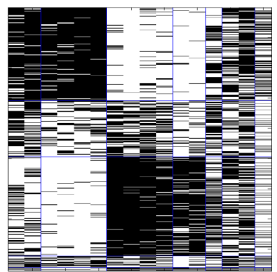

(c)

Fig. 2: Visualization of (a) the United State Congressional Voting Records data, where black cells indicate a "yea" and white cells represent a "nay"; (b) the same data matrix reorganized according to the partitions obtained with the NFLBM and (c) reorganized according the partition of LBM (Keribin et al, 2014). Irrelevant features are isolated on the left side cluster delimited by the red line. Blue lines indicate the relevant block structure.

political affiliation. Furthermore, our procedure separates the greatest non-voters, who chose to abstain from voting on more than $50 \%$ of the laws (cluster 1). Finally, in terms of variable cluster, the last political issue is not considered as being part of the noise cluster, and constitute a cluster by itself, because the non-voters all abstained on this one. In comparison, Keribin et al (2014) reported a partition into $5 \times 7$ clusters (according to the ICL) with the classic LBM. The main difference lies in the fact that the LBM created supplementary variable clusters for the laws identified as noise by the NFLBM. Also, we obtain a smaller number of cluster (both on the members and the laws) than Wyse and Friel (2012).

\subsection{Gibbs sampling for the binary NFLBM}

Hereafter, we propose to derive a second algorithm from our model, based on Gibbs sampling. The Gibbs sampler and the V-Bayes algorithms are complementary: the first one uses a single Markov Chain, and as a result is less sensitive to initialization; however, the returned estimator is close to the maximum without necessarily being the maximum. On the other hand, V-Bayes is more sensitive to initialization but guarantees that the obtained estimator corresponds to a local maximum. Therefore, coupling the two increases the chances of having the maximum likelihood estimator.

As $\boldsymbol{\theta}$, the set of parameters, is assumed to be a random variable, Gibbs sampling aims to estimate the distribution $p(\mathbf{x}, \mathbf{z}, \mathbf{w}, \boldsymbol{\theta})$ with a Monte Carlo Markov Chain. Therefore, the idea is to simulate each of the parameter according to a conditional probability of the others:

1. Simulation of $\mathbf{z}^{(c+1)}$ according to $p\left(\mathbf{z} \mid \mathbf{x}, \mathbf{w}^{(c)}, \boldsymbol{\theta}^{(c)}\right)$ defined by:

$$
p\left(\mathbf{z} \mid \mathbf{x}, \mathbf{w}^{(c)}, \boldsymbol{\theta}^{(c)}\right)=\prod_{i=1}^{n} \mathcal{M}\left(1 ; s_{i 1}, \ldots, s_{i g}\right)
$$

where $s_{i k}$ is defined by Equation (2).

2. Simulation of $\mathbf{w}^{(c+1)}$ according to $p\left(\mathbf{w} \mid \mathbf{x}, \mathbf{z}^{(c+1)}, \boldsymbol{\theta}^{(c)}\right)$ defined by:

$$
p\left(\mathbf{w} \mid \mathbf{x}, \mathbf{z}^{(c)}, \boldsymbol{\theta}^{(c)}\right)=\prod_{j=1}^{d} \mathcal{M}\left(1 ; t_{j 0}, t_{j 1}, \ldots, t_{j m}\right)
$$


where $t_{j \ell}$ is defined by Equations (3) and (4).

3. Simulation of $\boldsymbol{\pi}^{(c+1)}$ according to $\operatorname{Dir}\left(a+z_{+1}^{(c+1)}, \ldots, a+z_{+g}^{(c+1)}\right)$.

4. Simulation of $\boldsymbol{\tau}^{(c+1)}$ according to $\operatorname{Dir}\left(a+w_{+1}^{(c+1)}, \ldots, a+w_{+m}^{(c+1)}\right)$.

5. Simulation of $\phi^{(c+1)}$ according to $\mathcal{B} e\left(c_{1}+d-w_{+0}^{(c+1)}, c_{2}+w_{+0}^{(c+1)}\right)$.

6. Simulation of $\lambda_{j}^{(c+1)}$ for any $j$ such that $w_{j 0}=1$ according to $\mathcal{B} e\left(e_{1}+x_{+j}, e_{2}+n\right)$.

7. Simulation of $\alpha_{k \ell}^{(c+1)}$ according to

$$
\prod_{k=1}^{g} \prod_{\ell=1}^{m} \mathcal{B} e\left(\sum_{i=1}^{n} \sum_{j=1}^{d} z_{i k}^{(c+1)} w_{j \ell}^{(c+1)} x_{i j}, z_{+k}^{(c+1)} w_{+\ell}^{(c+1)}\right) .
$$

The main goal of Gibbs sampling is to create a Markov chain that will give an overview of the posterior distribution of $(\mathbf{z}, \mathbf{w}, \boldsymbol{\theta})$. This overview relies on the idea that, as the number of iteration tends to infinity, the more likely a region is, the more parameters they will be in this specific region. One should also note, that the distribution has a great number of symmetries, due to the problem of label switching; for instance, if we switch two cluster numbers, let us say 1 and 2, the parameters will be different while they basically represent the same cluster. In order to solve this problem, we use the identifiability conditions given in Theorem 1 so as to order the clusters, then we average all simulated $\boldsymbol{\theta}$ 's, and the assignment to a specific cluster is done according to a simple majority vote.

\section{Model selection}

Clustering, and by extension co-clustering, poses the question of defining an appropriate number of (co)-clusters. For mixture model, this problem is generally framed as a problem of model selection, and remains a difficult challenge, as choosing the best model according to the maximum likelihood usually leads to pick the most complex one. To overcome this issue, a popular approach is to penalize the likelihood of the model by, for instance, the number of parameters. For the latent block models, two common criteria have been adapted: the Bayesian Information Criterion (BIC) which is known to be consistent, and the Integrated Completed Likelihood (ICL) (Keribin et al, 2014; Wyse et al, 2017), that aims to minimize the entropy of $\mathbf{z}$ and $\mathbf{w}$. In the context of the LBM, empirical studies carried out by Keribin et al (2014) show that the two estimates provided by each of the criteria are asymptotically equal. For the NFLBM, in case of binary data with the conjugate prior that we chose in the previous section, the ICL can be written as

$$
\begin{aligned}
& \operatorname{ICL}(g, m) \\
& =\max _{(\mathbf{z}, \mathbf{w}) \in \mathcal{Z} \times \mathcal{W}} p(\mathbf{x}, \mathbf{z}, \mathbf{w} ; g, m) \\
& =\max _{(\mathbf{z}, \mathbf{w}) \in \mathcal{Z} \times \mathcal{W}}\left\{\log \Gamma(g a)-g \log \Gamma(a)-\log \Gamma(n+g a)+\sum_{k=1}^{g} \log \Gamma\left(z_{+k}+a\right)\right. \\
& \quad+\log \Gamma(m a)-m \log \Gamma(a)+g m(\log \Gamma(2 b)-2 \log \Gamma(b))+\sum_{\ell=1}^{m} \log \Gamma\left(w_{+\ell}+a\right) \\
& \quad-\log \Gamma\left(d-w_{+0}+m a\right)+w_{+0}\left[\log \Gamma\left(e_{1}+e_{2}\right)-\log \Gamma\left(n+e_{1}+e_{2}\right)-\log \Gamma\left(e_{1}\right)\right.
\end{aligned}
$$




$$
\begin{aligned}
& \left.-\log \Gamma\left(e_{2}\right)\right]+\sum_{j=1}^{d} w_{j 0}\left[\log \Gamma\left(x_{+j}+e_{1}\right)+\log \Gamma\left(n-x_{+j}+e_{2}\right)\right]+\log \Gamma\left(c_{1}+c_{2}\right) \\
& -\log \Gamma\left(c_{1}\right)-\log \Gamma\left(c_{2}\right)+\log \Gamma\left(d-w_{+0}+c_{1}\right)+\log \Gamma\left(w_{+0}+c_{2}\right)-\log \Gamma\left(d+c_{1}+c_{2}\right) \\
& \left.+\sum_{k=1}^{g} \sum_{\ell=1}^{m}\left[\log \Gamma\left(N_{k \ell}^{\mathbf{z w}}+b\right)+\log \Gamma\left(z_{+k} w_{+\ell}-N_{k \ell}^{\mathbf{z w}}+b\right)-\log \Gamma\left(z_{+k} w_{+\ell}+2 b\right)\right]\right\} \text { (7) }
\end{aligned}
$$

for each pair $(g, m)$, where $\Gamma$ is the Gamma function such that $\Gamma(n+1)=n !, N_{k \ell}^{\mathbf{z w}}=$ $\sum_{i=1}^{n} \sum_{j=1}^{d} z_{i k} w_{j \ell} x_{i j}$, and $\mathbf{z}$ and $\mathbf{w}$ are estimated by the algorithm. The calculations are derived from the appendix of Keribin et al (2014). Orange and red parts correspond to the elements which are not present in the ICL for the classic LBM. When the noise cluster is empty, the orange part is equal to zero; in this case, our criteria becomes equivalent to the one of LBM if we assume Dirac distribution instead of the Beta prior for the parameter $\phi$.

In this work, we choose the ICL for three reasons: (1) as the ICL criterion aims at minimizing the entropy of the partitions, it is known to give more homogeneous clusters than other criteria (see Baudry et al (2008)); (2) the conjecture given in Section 4.2 by Keribin et al (2014) suggests that, for the LBM, both the BIC and the ICL have the same asymptotic behavior; (3) finally, the ICL is exact at finite distance and relies on the same Bayesian assumptions as the ones used in our model (V-Bayes and Gibbs).

\section{Theoretical Analysis}

In this section, we propose to study the theoretical properties of the NFLBM. We start by showing that under reasonable assumptions, the model is identifiable, and proceed with results on its consistency.

Theorem 1 (Identifiability) Consider the binary NFLBM with $\boldsymbol{\pi}$ and $\boldsymbol{\tau}$ be the row and column mixing proportions and $\boldsymbol{\alpha}=\left(\alpha_{k, \ell}\right)$ the $g \times m$ matrix of Bernoulli parameters. The binary NFLBM is identifiable under one of these groups assumptions:

- If the true partition $\mathbf{w}_{., 0}^{\star}$ of the null columns cluster is known and:

- For all $k \in 1, \ldots, g, \pi_{k}>0$ and the coordinates of the vector $\boldsymbol{\alpha} \tau$ are distinct.

- For all $\ell \in 1, \ldots, m, \tau_{\ell}>0$ and the coordinates of the vector $\boldsymbol{\pi}^{\prime} \boldsymbol{\alpha}$ are distinct.

- $n \geq 2 m-1$ and $d-w_{+0}^{\star} \geq 2 g-1$.

- If every $\lambda_{j}=\lambda$ and

- For all $k \in 1, \ldots, g, \pi_{k}>0$ and the coordinates of the vector $\phi \boldsymbol{\alpha} \tau$ are distinct and distinct of $(1-\phi) \lambda$.

- For all $\ell \in 1, \ldots, m, \tau_{\ell}>0$ and the coordinates of the vector $\boldsymbol{\pi}^{\prime} \boldsymbol{\alpha}$ are distinct and distinct of $\lambda$.

- $n \geq 2 m-1$ and $d \geq 2 g+1$.

Proof For the first conditions, the key of the proof is in two steps: (1) if the column $j$ is in the cluster 0 , the identifiability is guaranteed by the identifiability of the Bernoulli distribution; (2) for the other columns, the identifiability is a corollary of the result of the latent block model (see Keribin et al (2014)). For the second condition, one can observe that we simply have a LBM where the first cluster has $g$ times the same value, and, therefore we are in the conditions of Keribin et al (2014).

Now, we give result on the consistency of the estimators obtained in NFLBM. 
Theorem 2 (Consistency) For the consistency, we need some assumptions on the parameters space $\Theta$ :

$\mathcal{A}_{1} \quad$ There exists a positive constant $\delta$ such that the space of parameters of $\left(\pi^{\star}, \phi^{\star}, \tau^{\star}\right)$ is included in $[\delta ; 1-\delta]^{g+1+m+g \times m}$.

$\mathcal{A}_{2}$ The true set of parameter $\boldsymbol{\theta}^{\star}=\left(\boldsymbol{\pi}^{\star}, \phi^{\star}, \boldsymbol{\tau}^{\star}\right)$ lies in the relative interior of the previous part of $\boldsymbol{\Theta}$.

$\mathcal{A}_{3} \quad$ All rows and columns of $\boldsymbol{\alpha}^{\star}$ are unique.

$\mathcal{A}_{4 . a}$ These both assumptions are true:

- Every $\lambda_{j}^{\star}$ equal to the same $\left.\lambda^{\star} \in\right] \delta ; 1-\delta[$.

- No column of $\boldsymbol{\alpha}^{\star}$ is constant equal at $\lambda$.

$\mathcal{A}_{4 . b}$ These both assumptions are true:

- The number $w_{+0}^{\star}$ of the noises columns is known.

- There exists $\gamma>0$ such that for every $\lambda_{j}$ and $(k, \ell) \in\{1, \ldots, g\} \times\{1, \ldots, m\}$, we have: $\left|\lambda_{j}-\alpha_{k \ell}\right|>\delta$.

Under the assumptions $\mathcal{A}_{1}, \mathcal{A}_{2}, \mathcal{A}_{3}$ and $\left(\mathcal{A}_{4 . a}\right.$ or $\left.\mathcal{A}_{4 . b}\right)$ the estimator of the maximum likelihood is consistent (up to one permutation of the cluster number).

Proof With the assumption $\mathcal{A}_{4 . a}$, the model can be seen as a classic LBM under the assumption of Brault et al (2017). The sketch of the proof with $\mathcal{A}_{4 . b}$ is that if the number of columns is fixed and the distance between the parameters is large enough, then the classification between noise or relevant is consistent and the rest is under the conditions of the estimation of the LBM.

\section{Experimental Results}

In order to give a comprehensive evaluation of the proposed models, we first conduct extensive experimentation on synthetic binary datasets, then demonstrate their efficiency on real DNA sequence datasets. In what follows, posterior probabilities returned by all approaches (see Equations 2, 3 and 4) are converted into crisp partitions by using the Maximum A Posteriori (MAP) principle. Both the implementation of the model and the pre-processed real datasets can be found online ${ }^{2}$.

\subsection{Synthetic data}

Setting. Monte-Carlo experiments are performed on $(n \times d)$, with $n \in\{100,1000\}$ and $d \in\{60,600\}$, synthetic binary datasets, arising from a $(g, m)=(5,3)$ block structure with equal proportions in order to compare the behaviour of the proposed algorithm. The simulation process fits the generative process described previously and we consider three scenarios differing in the percentage of irrelevant features (sampled from a $\mathcal{B}(\lambda), \lambda$ being randomly drawn) introduced: $10 \%$ (i.e., $\phi=0.9$ ), $50 \%$ (i.e., $\phi=0.5$ ) and $80 \%$ (i.e., $\phi=0.2)$ successively. In addition, we consider easily $[+](\varepsilon=0.25)$, moderately [++] $(\varepsilon=0.35)$ or hardly $[+++](\varepsilon=0.45)$ separated mixtures, where $1-2 \varepsilon$ corresponds to the minimum distance between the rows and the columns of the $\alpha$ parameter.

\footnotetext{
2 The datasets can be found here: https://github.com/laclauc/NFLB and the code will be available upon publication.
} 

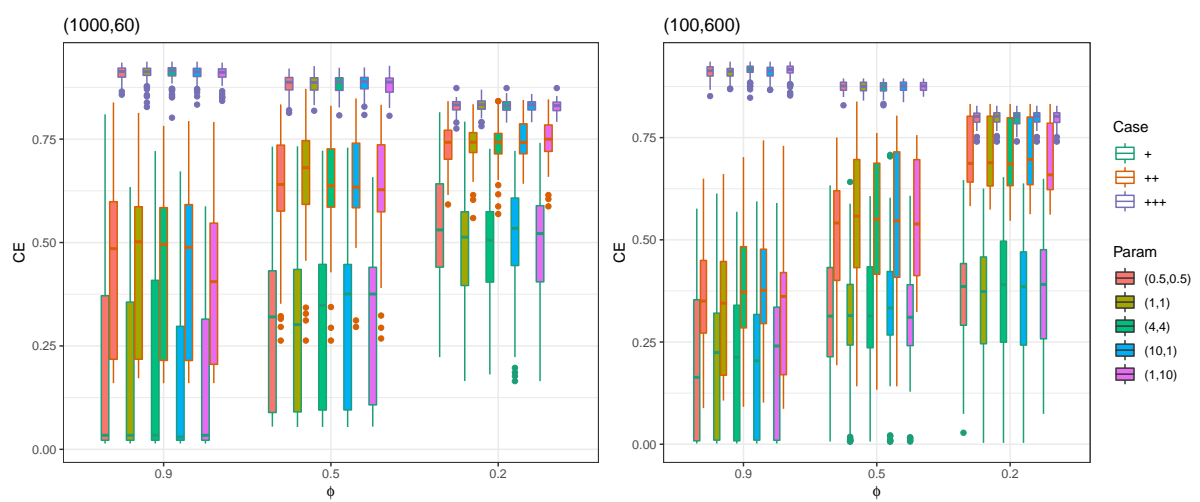

Fig. 3: CE obtained by V-Bayes as a function of the hyper-parameters $c_{1}$ and $c_{2}$ (color inside the boxplots), the dimension of the data (left vs. right), $\phi$ (x-axis on the plots) and the degree of overlapping between co-clusters (color legend outside the boxplots).

We compare B-NFLBM, referred to as V-Bayes and its Gibbs version, referred to as Gibbs in the results in terms of two metrics: the co-clustering error (CE) (Patrikainen and Meila, 2006) and the co-clustering extension of the Adjusted Rand Index (CARI) (Robert and Vasseur, 2017). The CE is defined as follows

$$
\mathrm{CE}((\mathbf{z}, \mathbf{w}),(\hat{\mathbf{z}}, \hat{\mathbf{w}}))=e(\mathbf{z}, \hat{\mathbf{z}})+e(\mathbf{w}, \hat{\mathbf{w}})-e(\mathbf{z}, \hat{\mathbf{z}}) \times e(\mathbf{w}, \hat{\mathbf{w}}),
$$

where $\hat{\mathbf{z}}$ and $\hat{\mathbf{w}}$ are the partitions of instances and variables estimated by the algorithm; $\mathbf{z}$ and $\mathbf{w}$ are the true partitions and $e(\mathbf{z}, \hat{\mathbf{z}})(\operatorname{resp} . e(\mathbf{w}, \hat{\mathbf{w}}))$ denotes the error rate, i.e., the proportion of misclassified objects (resp. features). The CE is in $[0,1], 0$ being the case where the partitions are the same. CARI, is a symmetric index and takes the value 1 when the couples of true and estimated partitions agree perfectly, up to a permutation. Finally, for all subsequent boxplot figures, whiskers correspond to $Q_{1}-1,5 I Q$ and $Q 3+1,5 I Q$, where $I Q$ is the interquartile range between $Q_{3}$ and $Q_{1}$.

Impact of hyper-parameters Hereafter, we study and discuss the impact of the different hyper-parameters involved in the NFLBM optimization. To proceed, we evaluate the performance of our approach in five different scenarios w.r.t. the values of $c_{1}$ and $c_{2}$, which are directly involved in the estimation of $\phi$.

1. $c_{1}=c_{2}=0.5$, corresponds to the case, where we consider non informative Jeffrey's prior, which tends to promote "all or nothing" type of models (i.e. all the variables are noise or none are);

2. $c_{1}=c_{2}=1$, corresponds to the case, where we use uniform distribution to avoid having any type of a priori information on the proportion of noise in the data;

3. $c_{1}=c_{2}=4$, corresponds to the case, where we assume that half of the variables are relevant (the other half being noise);

4. $c_{1}=1$ and $c_{2}=10$ is the case, where we assume that almost all variables are relevant;

5. $c_{1}=10$ and $c_{2}=1$ is the case where, we assume that almost all variables are irrelevant;

In Figure 3, we report the results obtained with V-Bayes only (as we obtain similar results with the Gibbs sampling) for the four aforementioned settings. One can observe that regardless the setting used to generate the data, the chosen couple $\left(c_{1}, c_{2}\right)$ is not impacting significantly the performance of the approach. For this reason, we propose to set $c_{1}=c_{2}=1$ 

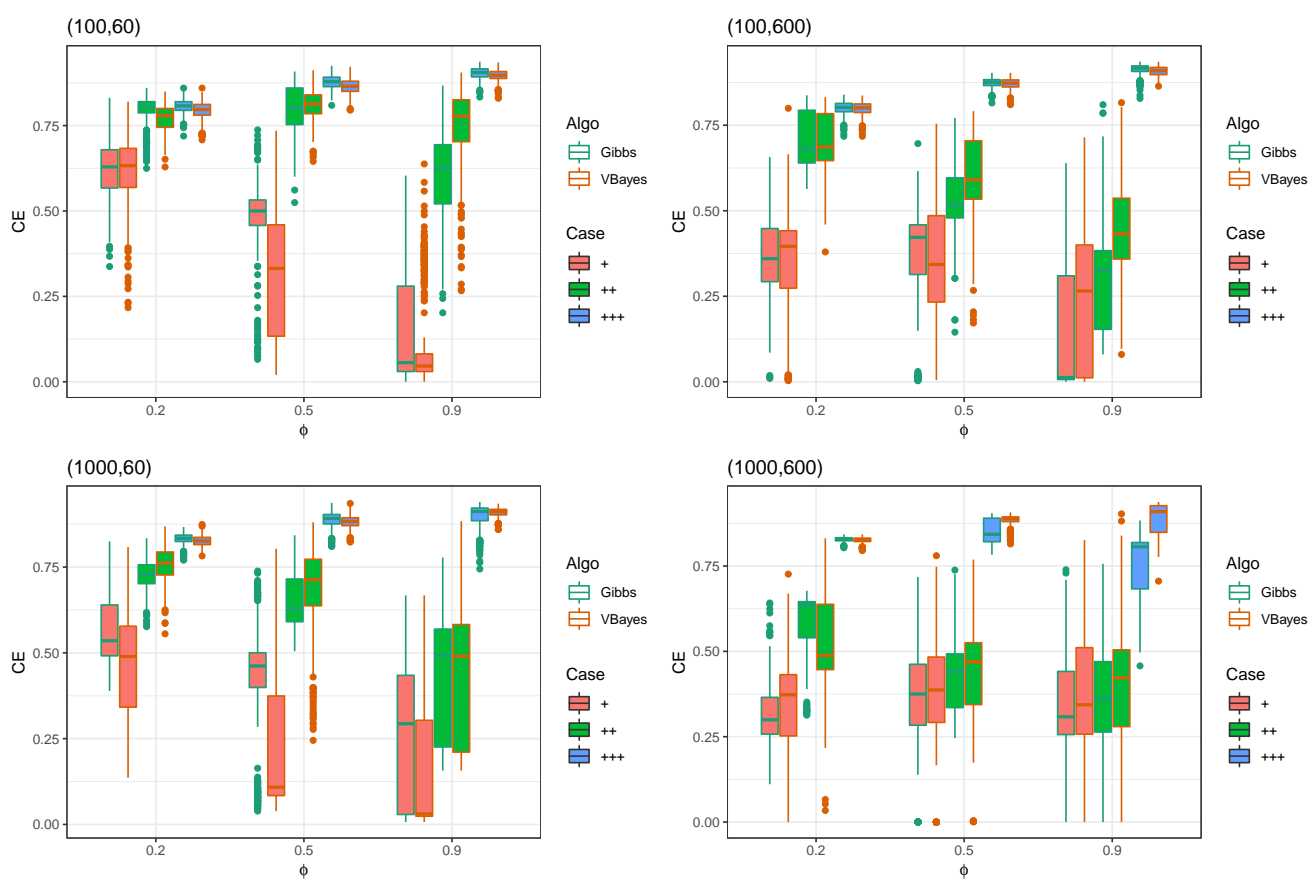

Fig. 4: $\mathrm{CE}$ on data matrices as a function of the number of rows and columns, $\phi$ (x-axis on the plots), the degree of overlapping between co-clusters (colors of the boxplots) and the chosen algorithms (outline color of the boxplots).

(no specific a priori information) for all experiments in the following. For other hyperparameters, we set $a=4, b=1$ as suggested by Keribin et al (2014). Finally, we set $e_{1}=e_{2}=1$, as we do not assume any a priori information on the values of $\boldsymbol{\lambda}$.

Estimating the partitions knowing $g$ and $m$. We compare the quality of the partitions $\mathbf{z}$ and w obtained with the V-Bayes algorithm and the V-Bayes with Gibbs sampling algorithm. For the first one, we randomly initialize the algorithm 10 times for each setting and keep the result that corresponds to the maximum likelihood. For the second one, we use 1000 iterations for the Gibbs sampling. In both cases, we set $g$ and $m$ to the values used during simulations. In the following, we only present the results in terms of CE for some of the settings as the results of CARI (and of other settings) were nearly the same. Figure 4 reports the results for different settings. One can observe that both algorithms give good performance in general and that none of them really seem to distinguish from the other. In addition, the quality of the estimation is inversely proportional to the difficulty of the data. To complete this analysis, we also provide a comparison between the models w.r.t. the estimation of the noise parameter $\phi$ (see Figure 5). First, we observe that both models tend to underestimate $\phi$, i.e., to overestimate the proportion of irrelevant features; for hardly separated mixtures $([+++])$, almost all the features are considered as being irrelevant. This phenomenon is simply the result of the fact that when generating a dataset with a block structure, we can only be sure that we will be able to retrieve the exact blocks if the number of observations is infinite. With a finite number of observations and especially in the case of hardly separable blocks, 

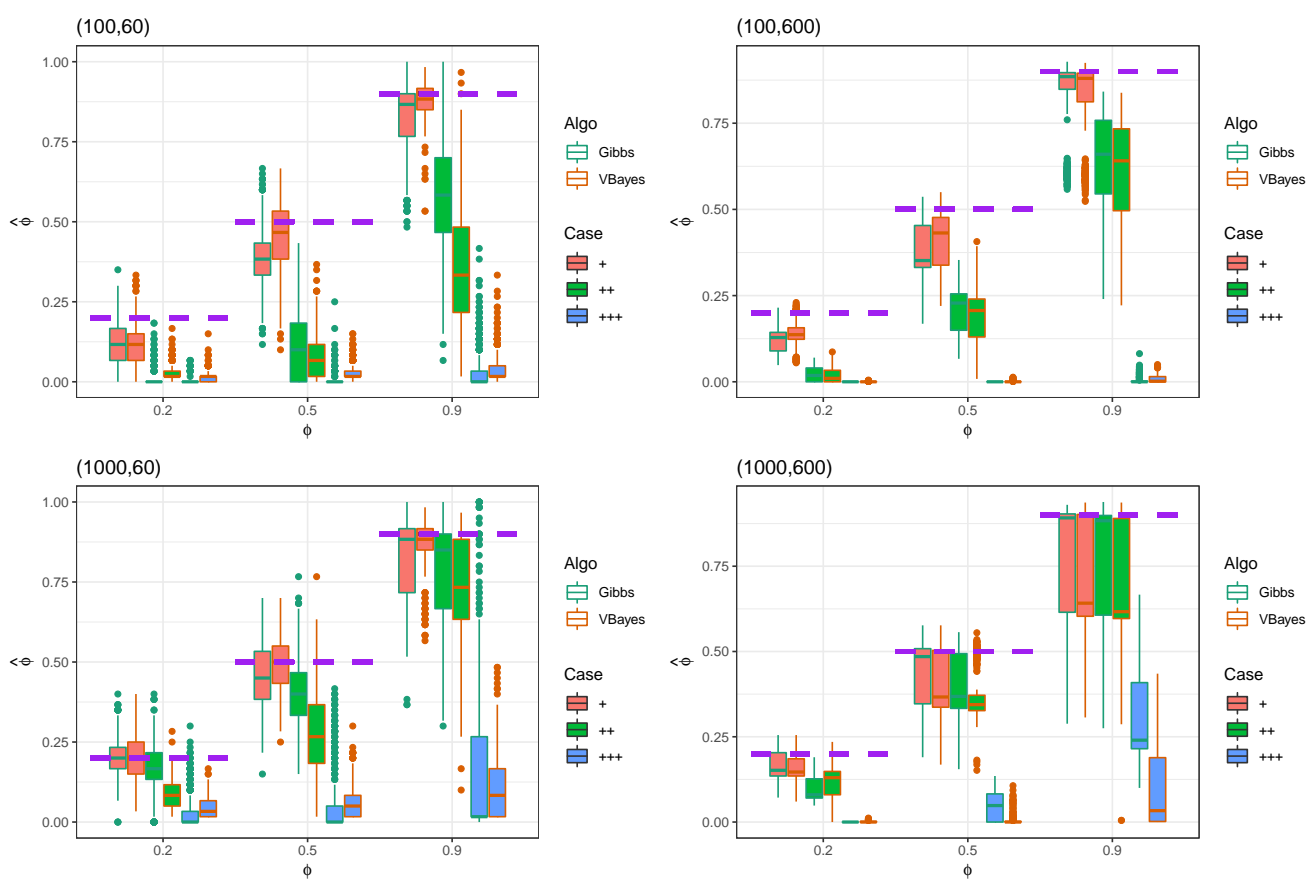

Fig. 5: Estimation of $\widehat{\phi}$ on data matrices as a function of the number of rows and columns, $\phi$ (x-axis on the plots), the degree of overlapping between co-clusters (colors of the boxplots) and the chosen algorithms (outline color of the boxplots). Purple dashed lines indicate the true values of $\phi$.

we can easily encounter co-clusters with a majority of features taking the value of 1's for instance, but which may also contain some features with 0's. These features, even though they were not originally simulated as being noisy, are therefore not relevant (and considered as such by NFLBM) for separating the clusters (see Figure 6).

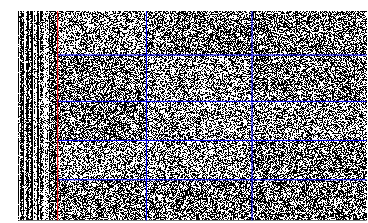

(a)

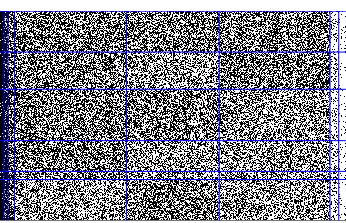

(b)

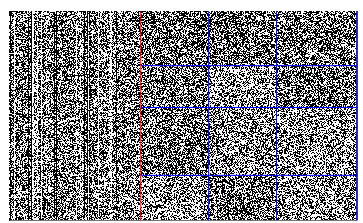

(c)

Fig. 6: Data matrix of size $(1000,600)$ with $\phi=0.9$ and ill-separated mixtures, reorganized according to : (a) the thrue partitions; (b) the partitions obtained with LBM; (c) the partitions obtained with NFLBM. 
Estimating $g$ and $m$. Next, we assess the ability of our approach to estimate the couple $(g, m)$ on the generated data. For each simulated matrix, we vary the number of row clusters between 2 and 8 and the number of column clusters between 1 and 6 . Table 1 reports the results obtained for $\phi=0.5$ (i.e. $50 \%$ of the relevant features) and [+] degree of separation. These results corresponds to the couple $(g, m)$ that maximizes the ICL criterion given in Equation 7.

Table 1: Frequency of the models selected by the ICL criterion on 100 data matrices with well separated clusters, and $50 \%$ of irrelevant features.

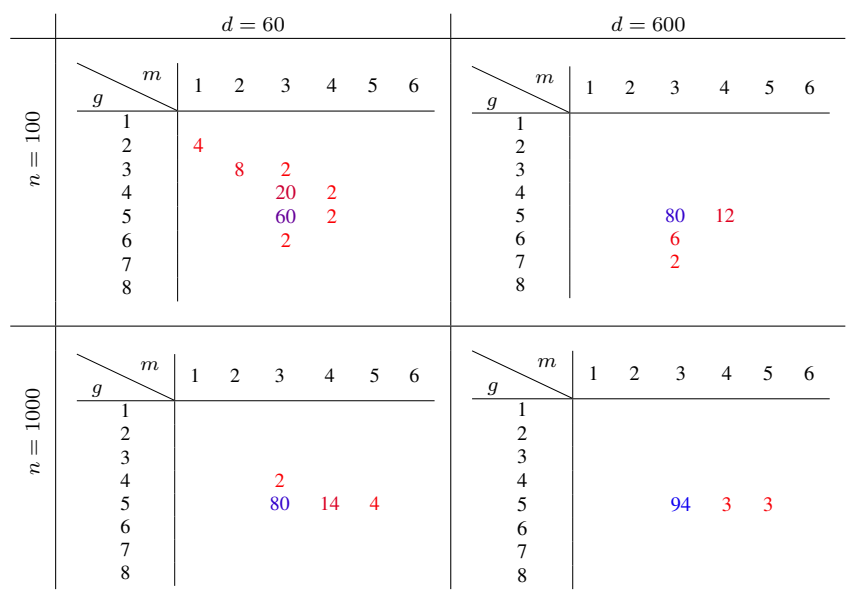

From this table, one can observe that in most cases, the ICL criterion correctly identifies both $g$ and $m$. We can also see that it tends to underestimate the number of clusters when the number of rows and columns are too small, and that the estimation gets better as the dimension of the data increases. In addition, one should note that when the number of rows is much greater than the number of columns $(n>d)$, ICL may overestimate the number of classes in columns; in contrast, if the number of columns is greater than the number of rows $(d>n)$, then $g$ can be slightly overestimated. In Table 2, we also report the results for the $1000 \times 600$ data with different levels of noise. One can see that the proposed approach is robust to the noise, as even with only $10 \%$ of relevant features $(\phi=0.1)$ the right couple $(g, m)$ is correctly identified in more than $95 \%$ of the cases. However, when $\phi$ is high (i.e., almost all the features are relevant), we observe we observe a behavior close to the one of LBM (Keribin et al, 2014), with a possible overestimation of the number of column clusters. This observation is coherent with the fact that when there is few noise, the NFLBM approaches the classic LBM.

NFLBM vs. LBM. Hereafter, we propose a strategy aiming to help the user in choosing between NFLBM, that aims to identify a noise cluster and LBM, which can be viewed as a specific case of NFLBM, where all variables are considered as relevant for the co-clustering. To proceed, we propose to compare both models on the basis of the ICL criterion defined previously. We use the exact same setting than for the other experiments, but set the number of clusters to their true values. While the derived criterion for both models provides a direct 
Table 2: Number of times (in \%) that NFLB identifies the correct $g$ and $m$ for $(n, d)=$ $(1000,600)$ and [+] datasets with different $\phi$.

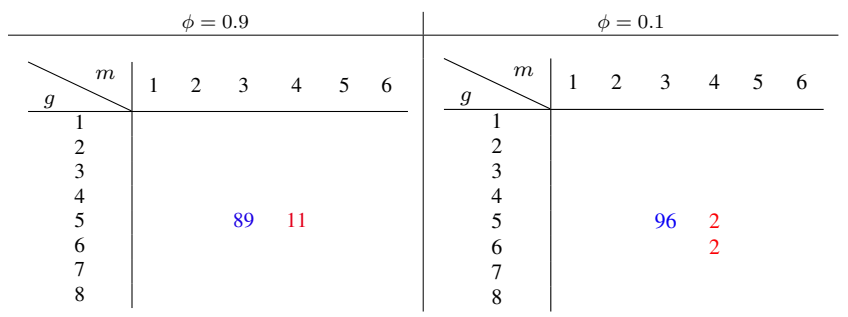

way to compare them, we would like to stress out a point, discussed in Section 5, related to the equivalence between these criterion. Indeed, we observed that the LBM criterion is close to that of the NFLBM if we assume a certain prior distribution over $\phi$ (and up to the red term in Equation 7). Now, the direct consequence is that if the noise component is empty, the ICL criterion of NFLBM is penalized by $\log (d+1)$ compared to that of LBM; this indicates that on equal partitions and in the absence of noise, the model selection procedure will always choose LBM over NFLBM. In order to highlight this point, we add a configuration with $\phi=1$ in the subsequent experiments.

Table 3 reports the number of times our model was selected over the classic LBM in all different settings. From these results, we can make three observations: (1) as expected, when $\phi=1$, LBM is almost always preferred to NFLBM; (2) on datasets with a high percentage of noise (i.e., a low value of $\phi$ ), the NFLBM is selected in most of the cases; (3) this assertion is all the more true when the dimension of the data and the number of observations increase. To this end, we believe that the proposed model is highly efficient in the situations that it was designed for, i.e. in dealing with high-dimensional noisy data.

Table 3: Number of times the NFLBM is chosen over the LBM over 100 trials. Here we consider different settings w.r.t. the percentage of noise $(\phi)$, the size of the data matix $(n, d)$ and the degree of overlap $([+],[++],[+++])$ between the blocks.

\begin{tabular}{|c|c|c|c|c|c|c|c|c|}
\hline & \multicolumn{4}{|c|}{$d=60$} & \multicolumn{4}{|c|}{$d=600$} \\
\hline @ & & + & ++ & +++ & & + & ++ & +++ \\
\hline$\|$ & 1 & 0 & 0 & 0 & 1 & 5 & 0 & 0 \\
\hline & 0.9 & 100 & 15 & 0 & 0.9 & 100 & 0 & 0 \\
\hline & 0.5 & 100 & 95 & 70 & 0.5 & 100 & 85 & 0 \\
\hline & 0.2 & 100 & 100 & 100 & 0.2 & 100 & 100 & 100 \\
\hline ఏ & & + & ++ & +++ & & + & ++ & +++ \\
\hline$\|$ & 1 & 0 & 0 & 30 & 1 & 0 & 0 & 10 \\
\hline & 0.9 & 100 & 100 & 50 & 0.9 & 100 & 100 & 85 \\
\hline & 0.5 & 100 & 100 & 100 & 0.5 & 100 & 100 & 100 \\
\hline & 0.2 & 100 & 100 & 100 & 0.2 & 100 & 100 & 100 \\
\hline
\end{tabular}

Finally, we also compare both models in terms of CE (see Figure 7). We observe that for the noisy configurations $(\phi=0.2$ and $\phi=0.5$ ), NFLBM significantly outperforms LBM. As for the case where $\phi=0.9$, we obtain better results with NFLBM when the 
mixtures are not too hardly separated $([+],[++])$, and comparable results on hardly separated mixtures with $\phi=1$. This result is quite interesting in its own right, but provides an even more powerful insight when combined to the observations made previously on Figure 5. Indeed, let us recall that when a large majority of the features are relevant, NFLBM tends to overestimate the size of the noise component, meaning that it chooses to exclude some potentially relevant features to obtain the partitions. However, despite this fact, NFLBM obtains comparable or better results in terms of co-clustering than LBM which considers all variables.
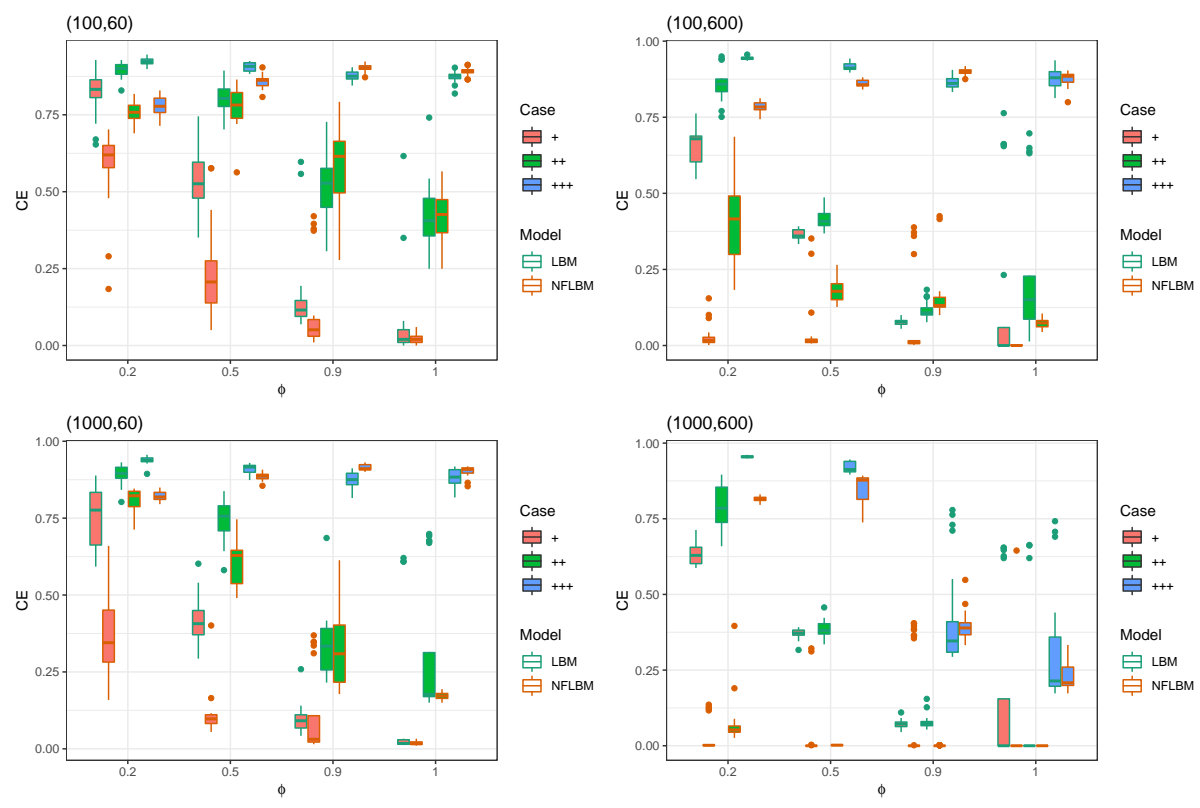

Fig. 7: CE on data matrices for the couple $(z, w)$ selected by the procedure as a function of the number of rows and columns, $\phi$ (x-axis on the plots), the degree of overlapping between co-clusters (colors of the boxplots) and the chosen model (outline color of the boxplots).

7.2 Genetic diversity through Microsatellites

A microsatellite is a DNA sequence formed by a continuous repetition of units usually composed of 1 to 4 nucleotides. The length of these sequences (i.e., the number of repeats) varies according to the species, but also from one individual to another and from one allele to the other. However, the location of these sequences in the genome is relatively similar between phylogenetically close species. In the following, we propose to apply the NFLBM to two microsatellite datasets, originally proposed to study the link between genetic diversity and geographical location of individuals. 
Description and pre-processing. The first dataset, referred to as DIVERSITY ${ }^{3}$, was proposed by Rosenberg et al (2002), in order to investigate genetic diversity and population structure in the world using genotypes at 377 autosomal microsatellite loci in 1056 individuals from 52 populations. The second one, referred to as NATIVE ${ }^{4}$ is a subset of the data reported by Wang et al (2007), which is an extension of DIVERSITY. The original dataset consists of 678 microsatellite loci genotyped in 1484 individuals from 78 worldwide populations including 29 Native American populations. In this work, we propose to focus on the Native American populations from north, central and south America, arising from 27 different tribes.

Pre-processing to obtain binary data is done as in the original papers. In addition, for the DIVERSITY data we propose to assess the capacity of our model to exploit only partial information. The original data contains genotypes measured in base pairs, and we propose to extract three biased versions: the two first versions only contain 1 out of the 2 pair and are referred to as DIVERSITY 1 and DIVERSITY 2 in the following. The third one, referred

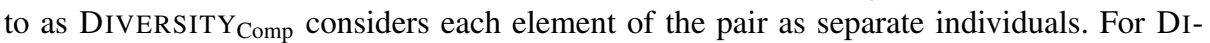
VERSITY $_{1}$ and DIVERSITY 2 the goal is to study if one of the elements of the base pair is more discriminant than the other for all individuals. Furthermore, it allows to evaluate the capacity of the model to recover a relevant partition of the individuals when considering half of the genetic information available. As for DIVERSITY ${ }_{\text {Comp }}$, we intend to demonstrate that our approach is able to capture the correlation between the elements of each pair. This setting provides a way to evaluate the quality of the column partitioning, which is usually more difficult to assess (see Table 6).

Table 4 provides details on all datasets. Finally, for all datasets, we select the number of clusters based on the ICL criteria.

Table 4: Properties of the different pre-processed datasets.

\begin{tabular}{ccccc}
\hline Properties & DIVERSITY $_{\text {Comp }}$ & DIVERSITY $_{1}$ & DIVERSITY $_{2}$ & NATIVE \\
\hline$n$ & 2112 & 1056 & 1056 & 494 \\
$d$ & 4689 & 3949 & 3867 & 5709 \\
Sparsity & $92.2 \%$ & $90.8 \%$ & $90.6 \%$ & $88.1 \%$ \\
\hline
\end{tabular}

Results. Table 5 presents the best ICL of both models, the estimated number of clusters, as well as the proportion of variables denoted by $\widehat{\phi}$. Regarding the model selection, we see that, as expected, the ICL favors LBM over NFLBM. However, despite the bias explained in the

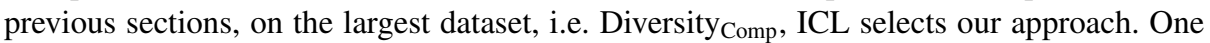
also observe that when we only take into account partial information, i.e., one of the two elements of the pair, (DIVERSITY 1 and DIVERSITY 2 ), then only around one third on the features are considered as relevant, while for the full data, the model is more conservative and identify twice this proportion as relevant. For the NATIVE data, $85 \%$ of the features are clustered as irrelevant. This is in line with the fact that this dataset only studies individuals from the same continent, who have more characteristics in common (i.e. non discriminant). From this dataset, we also observe two interesting phenomenon: (1) our model separate all the tribes which were added to the data by Wang et al (2007). This might be the results of a

\footnotetext{
3 https://rosenberglab. stanford.edu/data/rosenbergEtAl2002/ diversitydata.stru

4 https://rosenberglab.stanford.edu/nativedata.html
} 
different coding of the newly gathered data. (2) The 19 clusters are usually associated to one or two tribes. For the comparison with LBM, one can observe that LBM tends to estimate significantly more column clusters, when compared to NFLBM. As a result, the model also tends to merge row clusters, corresponding in this case to either continents or tribes, making them less meaningful and more difficult to interpret. Finally, Figure 8 shows one of the data matrix reorganized according to the partitions obtained with both models, and confirms our previous comments.

Table 5: Values of the ICL criterion and parameters estimated by NFLBM and LBM on all four datasets: number of row and column clusters $(\widehat{g}, \widehat{m})$, proportion of relevant features $(\widehat{\phi})$.

\begin{tabular}{|c|c|c|c|c|}
\hline Estimation & DIVERSITY $_{\text {Comp }}$ & DIVERSITY $_{1}$ & DIVERSITY $_{2}$ & NATIVE \\
\hline \multicolumn{5}{|l|}{ NFLBM } \\
\hline ICL & -1904961 & -965601.2 & -917814.9 & -710209.8 \\
\hline$\widehat{g}$ & 16 & 4 & 6 & 19 \\
\hline$\widehat{m}$ & 49 & 32 & 26 & 27 \\
\hline$\widehat{\phi}$ & $66.9 \%$ & $31.7 \%$ & $37.7 \%$ & $14.4 \%$ \\
\hline \multicolumn{5}{|l|}{ LBM } \\
\hline ICL & -1910703 & -959324.7 & -910975.5 & -696490.4 \\
\hline$\widehat{g}$ & 3 & 4 & 4 & 13 \\
\hline$\widehat{m}$ & 78 & 47 & 55 & 51 \\
\hline
\end{tabular}
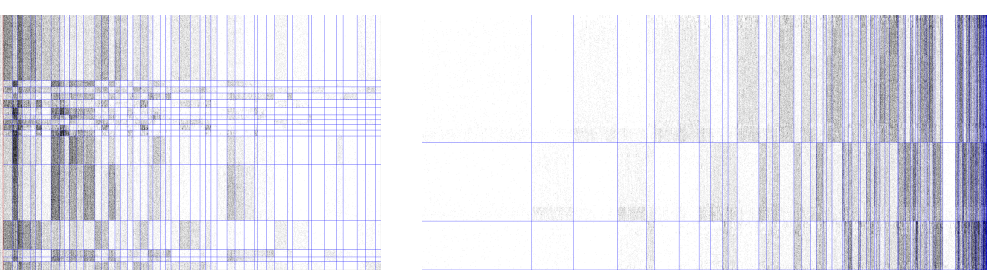

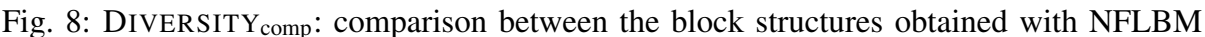
$(g, m)=(16,49)$, on the left and LBM $(g, m)=(3,78)$, one the right. For NFLBM, the noise component is delimited by the red line.

Figure 9 shows the overlap between the partition of observations and the continent information. In both cases, we note that four of the clusters corresponds exactly to 4 of the continents. However, the three remaining ones are together in one cluster; for instance, Europe with Central, South Asia. We see two possible explanations: (1) there exist a wellknown proximity between some countries from the different continents (e.g Russia is part of Europe but geographically close to central Asia); (2) we only use half of the genotypes to characterize the individuals.

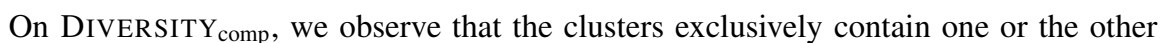
element in the pair (explaining also the higher number of clusters). However, we also see that the clusters are slightly different from the agglomeration of partitions from DIVERSITY 1 and DIVERSITY 2 . We have 16 clusters divided into 7 clusters for the first information and 9 clusters for the second one, against 4 and 6 for DIVERSITY 1 and DIVERSITY 2 , respectively. Table 6 presents the number of individuals belonging to the same pair of clusters. One can see that to each cluster of the first element in the pair we can associate one cluster build with the second element. This result shows that NFLBM is able to capture the correlation 

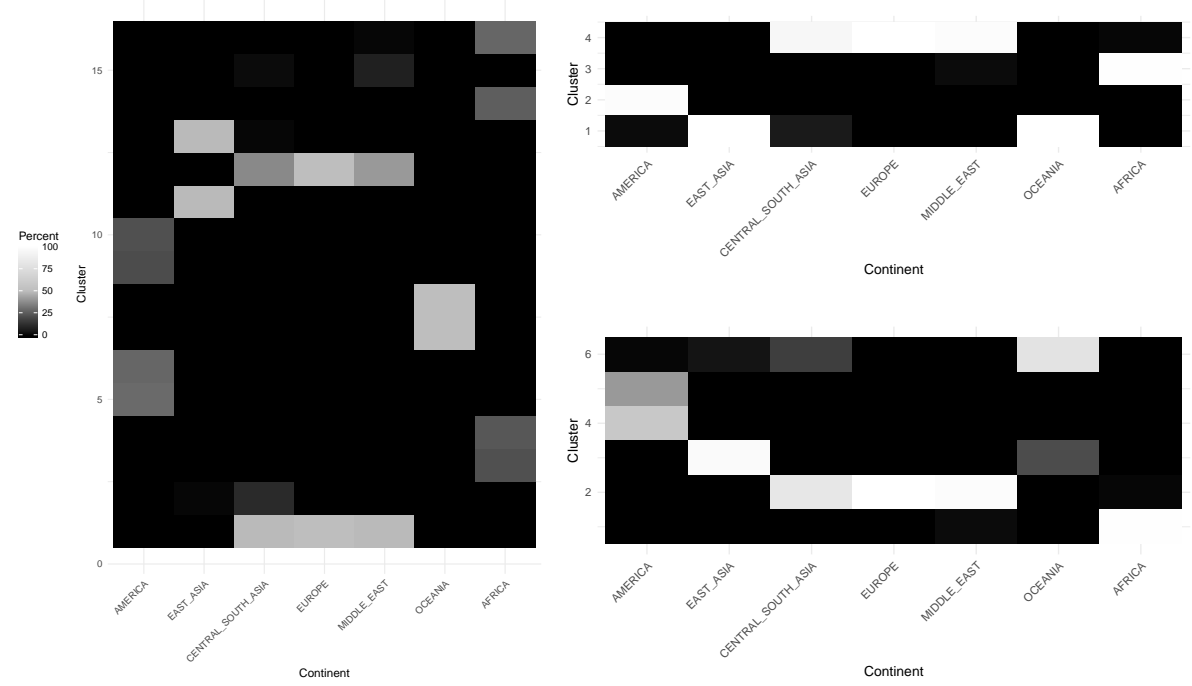

Fig. 9: Proportion of the observations from a continent that fall in each row cluster for the DIVERSITY datasets: DIVERSITY Comp $_{\text {(left), DIVERSITY }}$ (top right) and DIVERSITY 2 (bottom right).

between two elements of the same pair, as one can observe that the individuals are somehow implicitly clustered on the basis of the pair and not on the basis of each element separately.

Table 6: Number of individuals present in each cluster of the first and second elements of the pair.

\begin{tabular}{c|ccccccccc} 
& 2 & 4 & 6 & 7 & 10 & 11 & 12 & 14 & 15 \\
\hline 1 & 45 & & & & & & 464 & & 34 \\
3 & & 48 & & & & & & 4 & \\
5 & & & 59 & & 5 & & & & \\
8 & 2 & & & 39 & & 1 & & & \\
9 & & & 2 & & 43 & & & & \\
13 & 5 & & & & & 235 & & 59 & 3 \\
16 & & 8 & & & & & &
\end{tabular}

\section{Conclusion}

In this article, we proposed to study the problem of joint co-clustering and feature selection using the framework of mixture models. To this end, we proposed a new framework that states the existence of a variable noise cluster, allowing a flexible definition of a noisy feature. From this framework, we derived two Bayesian models and adapted the ICL criteria for selecting an appropriate number of clusters. We first validated our approach on synthetic data and then applied it on a real-world application where the goal is to explore genetic diversity across the world. We were able to show the interest of feature selection in order to maintain good clustering results in presence of noise. 
Although the results obtained with this new framework are promising, it admits some further improvements. From a theoretical point of view, the next step would be to analyze the properties of robustness of NFLBM. We believe that this type of study will allow us to better understand which model, between LBM and NFLBM is best suited for data generated differently (for instance, with overlap). It will also complete the strategy based on the ICL for choosing between these models. Now, from an algorithmic point of view, we would like to extend our approach to stochastic variational inference (Hoffman et al, 2013), that is a scalable algorithm for approximating posterior distributions. On the other hand, it might be interesting to impose a penalty term on the number of variables contained in the noise cluster.

\section{References}

Baudry JP, Celeux G, Marin JM (2008) Selecting models focussing on the modeller's purpose. In: COMPSTAT 2008, Springer, pp 337-348

Ben-David S, Haghtalab N (2014) Clustering in the presence of background noise. In: Proceedings of ICML, pp 280-288

Biernacki C, Celeux G, Govaert G (2000) Assessing a mixture model for clustering with the integrated completed likelihood. PAMI 22(7):719-725

Bouveyron C, Brunet-Saumard C (2014) Model-based clustering of high-dimensional data: A review. Computational Statistics \& Data Analysis 71:52-78

Brault V, Keribin C, Mariadassou M (2017) Consistency and asymptotic normality of latent blocks model estimators. arXiv preprint arXiv:170406629

Celeux G, Martin-Magniette ML, Maugis C, Raftery AE (2011) Letter to the editor: "A framework for feature selection in clustering". Journal of the American Statistical Association 106:383

Cuesta-Albertos JA, Gordaliza A, Matràn C (1997) Trimmed $k$-means: an attempt to robustify quantizers. The Annals of Statistics 25(2):553-576

Dave RN (1991) Characterization and detection of noise in clustering. Pattern Recogn Lett 12(11):657-664

Dave RN (1993) Robust fuzzy clustering algorithms. In: [Proceedings 1993] Second IEEE International Conference on Fuzzy Systems, pp 1281-1286 vol.2

Ester M, Kriegel HP, Sander J, Xu X (1996) A density-based algorithm for discovering clusters a density-based algorithm for discovering clusters in large spatial databases with noise. In: Proceedings of KDD, AAAI Press, pp 226-231

Frühwirth-Schnatter S (2011) Dealing with label switching under model uncertainty. In: Mixtures: Estimation and Applications, Wiley, chap 10, pp 213-239

García-Escudero LA, Gordaliza A, Matrán C, Mayo-Iscar A (2008) A general trimming approach to robust cluster analysis. The Annals of Statistics 36(3):1324-1345

García-Escudero LA, Gordaliza A, Matrán C, Mayo-Iscar A (2010) A review of robust clustering methods. Advances in Data Analysis and Classification 4(2):89-109

Govaert G, Nadif M (2003) Clustering with block mixture models. Pattern Recognition 36:463-473

Govaert G, Nadif M (2008) Block clustering with bernoulli mixture models: Comparison of different approaches. Computational Statistics \& Data Analysis 52(6):3233 - 3245

Govaert G, Nadif M (2013) Co-clustering. Wiley Online Library

Hartigan JA (1972) Direct Clustering of a Data Matrix. Journal of the American Statistical Association 67(337):123-129 
Hoffman MD, Blei DM, Wang C, Paisley J (2013) Stochastic variational inference. J Mach Learn Res 14(1):1303-1347

Keribin C, Brault V, Celeux G, Govaert G (2014) Estimation and selection for the latent block model on categorical data. Statistics and Computing pp 1-16

Law MHC, Figueiredo MAT, Jain AK (2004) Simultaneous feature selection and clustering using mixture models. IEEE Trans Pattern Anal Mach Intell 26:1154-1166

Li M, Zhang L (2008) Multinomial mixture model with feature selection for text clustering. Know-Based Syst 21(7):704-708

Maugis C, Celeux G, Martin-Magniette ML (2009) Variable selection for clustering with gaussian mixture models. Biometrics 65(3):701-709

Mirkin BG (1996) Mathematical classification and clustering. Nonconvex optimization and its applications, Kluwer academic publ, Dordrecht, Boston, London

Pan W, Shen X (2007) Penalized model-based clustering with application to variable selection. J Mach Learn Res 8:1145-1164

Patrikainen A, Meila M (2006) Comparing subspace clusterings. IEEE Transactions on Knowledge and Data Engineering 18(7):902-916

Raftery AE, Dean N (2006) Variable selection for model-based clustering. Journal of the American Statistical Association 101:168-178

Robert V, Vasseur Y (2017) Comparing high dimensional partitions, with the coclustering adjusted rand indew. CoRR abs/1705.06760

Rosenberg NA, Pritchard JK, Weber JL, Cann HM, Kidd KK, Zhivotovsky LA, Feldman MW (2002) Genetic structure of human populations. Science 298(5602):2381-2385

Wang S, Zhu J (2008) Variable selection for model-based high-dimensional clustering and its application to microarray data. Biometrics 64(2):440-448

Wang S, Lewis CM, Jakobsson M, Ramachandran S, Ray N, Bedoya G, Rojas W, Parra MV, Molina JA, Gallo C, Mazzotti G, Poletti G, Hill K, Hurtado AM, Labuda D, Klitz W, Barrantes R, Bortolini MC, Salzano FM, Petzl-Erler ML, Tsuneto LT, Llop E, Rothhammer F, Excoffier L, Feldman MW, Rosenberg NA, Ruiz-Linares A (2007) Genetic variation and population structure in native americans. PLoS Genetics 3(11)

Wang X, Kabán A (2005) Finding uninformative features in binary data. In: Intelligent Data Engineering and Automated Learning - IDEAL 2005, pp 40-47

Wyse J, Friel N (2012) Block clustering with collapsed latent block models. Statistics and Computing 22(2):415-428

Wyse J, Friel N, Latouche P (2017) Inferring structure in bipartite networks using the latent blockmodel and exact ICL. Network Science 5(1):45-69, DOI 10.1017/nws.2016.25, URL https: //doi.org/10.1017/nws.2016.25

Zhou H, Pan W, Shen X (2009) Penalized model-based clustering with unconstrained covariance matrices. Electronic Journal of Statistics 3:1473-1496 